\title{
MEC vs MCC: Análise do Desempenho de Aplicações Interativas e de Tempo Real
}

\author{
Micael Soares ${ }^{1}$, Pedro Pinto ${ }^{2,3,5}$, Jorge Mamede \\ 1000167@isep.ipp.pt, pedropinto@estg.ipvc.pt,jbm@isep.ipp.pt \\ ${ }^{1}$ Instituto Superior de Engenharia do Porto - Politécnico do Porto (ISEP-PP), Porto, Portugal \\ ${ }^{2}$ Instituto Politécnico de Viana do Castelo (IPVC), Viana do Castelo, Portugal \\ ${ }^{3}$ Instituto Universitário da Maia (ISMAI), Maia, Portugal \\ ${ }^{4}$ GILT, Porto, Portugal \\ 5 INESC-TEC, Porto, Portugal
}

DOI: 10.17013/risti.37.116-131

Resumo: A evolução das redes de telecomunicações tem promovido o desenvolvimento de novas aplicações para dispositivos móveis. Algumas destas aplicações exigem requisitos computacionais e energéticos que vão para além das capacidades dos dispositivos móveis. Neste contexto, pode ser utilizada a arquitetura Mobile Cloud Computing (MCC), que permite executar as aplicações em datacenters na cloud e aliviar o processamento nos dispositivos móveis. No entanto, algumas aplicações mais exigentes, e.g. interativas e de tempo real, são mais sensíveis ao atraso no processamento e comunicação da informação. Para estas aplicações, a arquitetura Mobile Edge Computing (MEC) pode ser utilizada como uma tecnologia intermédia que disponibiliza recursos computacionais e de armazenamento a partir da periferia da rede. Este artigo apresenta um estudo que avalia o desempenho das arquiteturas MCC e MEC na execução de duas aplicações tomadas como representativas do espectro das aplicações interativas, de tempo real e de processamento intensivo: o Fluid e o FaceSwap. São apresentados resultados que permitem quantificar o desempenho destas arquiteturas em diferentes circunstâncias.

Palavras-chave: Mobile, Cloud, Edge, Computing, Real-time, Interactive, Applications.

\section{MEC vs MCC: Performance Analysis of Interactive and Real-time Applications}

Abstract: Telecommunication networks evolution is driving the development of new applications for mobile devices. Some of these applications are resourceintensive and push computational and energy demands of mobile devices beyond the mobile hardware capabilities. In this context, Mobile Cloud Computing (MCC) architecture emerges as a solution for offloading mobile devices that allows to execute 
these applications in cloud datacenters thus reducing the processing demand in mobile devices. However, more demanding applications, e.g. interactive and realtime applications, are sensitive to processing and communications delay. For these applications, Mobile Edge Computing (MEC) can be used as an intermediary technology, providing computing and storage resources in the network edge. This paper presents a study carried out to evaluate the performance of MEC and MCC architectures when executing two applications, Fluid and FaceSwap, representative of real time and computing intensive applications. A set of scenarios were designed to quantify the performance of these architectures in different settings.

Keywords: Mobile, Edge, Cloud, Computing.

\section{Introdução}

Na última década, o Cloud Computing surgiu como um novo paradigma da ciência da computação, que fornece um conjunto de recursos partilhados e com alta disponibilidade, onde se incluem serviços centralizados de processamento, armazenamento e gestão, entre outros (Armbrust et al., 2009)(Zhang et al., 2010)(Araújo \& Cota, 2016). Estes serviços são disponibilizados aos utilizadores finais e podem ser utilizados por aplicações e dispositivos que geram informação e depois utilizam a cloud para armazenar e/ou processar esta informação. Conforme referido em (Fortes et al., 2016), os serviços de cloud computing têm grande potencial e os seus fornecedores poderão promover a sua adoção de modo a aumentar o reconhecimento e a notoriedade social destas soluções tecnológicas. O crescimento na adoção destes serviços permite responder ao previsível aumento dos dispositivos terminais dos utilizadores. Com paradigmas como a Internet of Things (IoT), prevê-se que a quantidade de dispositivos ligada a estes sistemas seja cada vez maior, assim como a quantidade de dados que exigem ser analisados, processados, transformados, armazenados e respondidos em tempo útil.

Em (Dinh et al., 2013) foi proposta a arquitetura Mobile Cloud Computing (MCC), que permite que os dados gerados pelos dispositivos móveis sejam transmitidos para a cloud com servidores centrais distribuídos geograficamente utilizados para armazenamento e processamento. Com esta arquitetura, à medida que a distância física entre os dispositivos móveis e os servidores aumenta, a latência de transmissão e o atraso de processamento também aumenta, o que pode comprometer a qualidade do serviço e experiência de utilização de algumas aplicações. Em (Qing et al., 2013) os autores propõem uma arquitectura, o CACTSE, para disponibilização de conteúdos a partir de cloudlets localizadas em servidores próximos dos equipamentos dos utilizadores e explorando a cooperação entre diversos terminais.

Para estas aplicações mais exigentes em termos de latência de comunicação e atraso de processamento, foi proposta uma arquitetura distribuída designada por Mobile Edge Computing (MEC) (Patel et al., 2014). Esta arquitetura é baseada na colocação de servidores na periferia da rede que fornece a capacidade computacional, analítica e de armazenamento, para que os dispositivos móveis tenham esforço computacional distribuído e menor latência e atraso.

Neste artigo apresenta-se uma análise comparativa do desempenho das arquiteturas MEC e MCC, na implementação de duas aplicações consideradas representativas 
das aplicações interativas, com requisitos de tempo real e processamento intensivo. São definidos cenários de teste que permitem analisar a influência da latência das comunicações e da capacidade de processamento dos servidores remotos em ambas as arquiteturas, a fim de quantificar o seu desempenho em várias vertentes.

Os resultados obtidos mostram de que forma o MEC pode melhorar a interação do utilizador e a qualidade da experiência, uma vez que coloca o processamento e a computação intensiva em servidores na periferia da rede, mais próximos dos dispositivos móveis.

Este artigo está estruturado em 5 secções. A primeira secção apresenta uma introdução ao trabalho realizado. Na Secção 2 apresentam-se trabalhos de outros autores relacionados com estudo reportado neste artigo. A Secção 3 descreve o cenário e os casos de teste das aplicações selecionadas. A Secção 4 apresenta os resultados obtidos e as respectivas análises. A secção 5 conclui este artigo.

\section{Trabalho Relacionado}

O cloud computing tem sido utilizado como forma de ultrapassar as limitações de processamento e de armazenamento dos sistemas computacionais na periferia da rede.

Em particular, a arquitetura MCC tem como objetivo dar resposta aos desafios de mobilidade dos equipamentos móveis e às suas limitações de recursos como o armazenamento, processamento e baterias (Beck et al., 2014). No que respeita ao tráfego que estes dispositivos móveis geram e comunicam, pela estimativa apresentada em (Blockstrand et al., 2011), até 2020 deverão existir 50 mil milhões de dispositivos inteligentes ligados à rede, com o volume de dados a crescer exponencialmente. De acordo com (Skarpness, 2017), em 2020 é esperada a geração de um tráfego de 1,5 GB de dados por pessoa por dia.

Porém, existem aplicações que, por um lado, produzem grandes quantidades de dados e necessitam de capacidade computacional, mas por outro, exigem interação com o utilizador e são sensíveis à latência e atraso. O potencial dos terminais tem estimulado o desenvolvimento de aplicações cada vez mais interativas, inteligentes e que exploram a mobilidade dos utilizadores tais como as aplicações de realidade aumentada, condução autónoma, ou de jogos em tempo real, que requerem uma conectividade permanente e com débitos de acesso elevados. Este tipo de aplicações pode não ser compatível com o MCC e, é neste cenário, que surgem as arquiteturas de Edge Computing (EC) (Verbelen et al., 2012) (Patel et al., 2014) (ETSI, 2014) (ETSI, 2015) (GSMA Intelligence, 2014) (Mao et al., 2017).

Nas arquiteturas de Edge Computing (EC) os servidores são colocados próximo da rede de acesso e dos utilizadores, e são dotados de recursos de processamento, armazenamento e de débito de acesso, que permitem executar as tarefas computacionais mais exigentes, retirando-as dos equipamentos terminais. Existem várias arquiteturas de implementação do EC como, por exemplo, o Fog Computing $(F C)$, as Cloudlets e o MEC (Mobile Edge Computing).

A arquitetura do FC foi introduzida pela Cisco em 2012 (Bonomi et al., 2012) e foi considerada uma extensão do paradigma da cloud que fornece serviços de computação, 
armazenamento e rede entre equipamentos terminais e os tradicionais servidores da cloud. O Open Fog Consortium definiu uma arquitetura distribuída de três camadas (utilizadores finais, nós de neblina e clouds centralizadas) em que os elementos comunicam e interagem entre si. O objetivo principal é aproximar os dados do utilizador final (Shenoy et al., 2015), o que reduz a latência, melhora a QoS (Suryawanshi \& Mandlik, 2015) e fornece suporte para localização, reconhecimento de contexto e suporte à mobilidade (Aazam \& Huh, 2014). O FC permite recolher dados e analisar a informação num contexto local, enquanto na cloud se coordena e realiza as análises globais para avaliação e satisfação dos requisitos das aplicações. Como a arquitetura $F C$ é heterogênea, os serviços podem ser instalados na periferia da rede em servidores em locais com débito elevado e acessíveis através de diferentes tecnologias sem fio (Bonomi et al., 2014).

A arquitetura Cloudlet foi desenvolvida por uma equipa da Universidade Carnegie Mellon (Satyanarayanan, 2009), que implementou um protótipo no âmbito do projeto Elijah (Elijah, 2009). Esta arquitetura assenta em três camadas compostas por dispositivos móveis, cloudlets e cloud. Nesta hierarquia, as cloudlets são a camada intermediária e consideradas como datacenters locais, que disponibilizam serviços de cloud localizados, e que proporcionam aos equipamentos dos utilizadores funcionalidades de alto desempenho computacional e de acesso mais rápido aos recursos da cloud (Klas, 2016) (Khan et al., 2014). As cloudlets são unidades servidoras projetadas para suportar aplicações de dispositivos móveis, executando as tarefas de computação intensiva de aplicações interativas, como, por exemplo, aplicações de tradução em tempo real (Achanta et al., 2012), aplicações de reconhecimento facial em tempo real (Soyata et al., 2012), aplicações de realidade aumentada (Abolfazli et al., 2013), jogos na cloud ou sistemas de assistência cognitiva. Nas cloudlets, as aplicações têm dois componentes principais, um que é executado no dispositivo móvel e outro na unidade servidora. Existem ainda outros trabalhos tal como (Koukoumidis et al., 2011) que propõem arquiteturas para cache dos serviços na cloud que são implementadas nos dispositivos móveis, utilizando uma pocket cloudlet.

Considerando as limitações computacionais e energéticas dos equipamentos terminais, a parte móvel da aplicação é a menos exigente em termos de recursos. Assim, é no servidor existente na cloudlet que se executam as tarefas da aplicação que necessitam de mais recursos computacionais e de armazenamento.

A filosofia da cloudlet foi seguida pelo European Telecommunications Standard Institute (ETSI), que entretanto normalizou a arquitetura MEC (Satyanarayanan, 2013) (Ai et al., 2017). A arquitetura do MEC é baseada numa plataforma de virtualização que permite a execução de aplicações em servidores virtuais colocados na periferia da rede de acesso, ou seja, pretende reduzir o tráfego da rede movendo os recursos da cloud para periferia da rede (Shahzadi et al., 2017), explorando de uma forma eficaz uma infraestrutura de sistemas totalmente virtualizada (Simanta et al., 2012). Esta infraestrutura pode ser implementada em servidores virtualizados colocados em locais diferentes, como pontos de acesso sem fio ( $A P$ - Access Points), em controladores de rede de rádio $(R N C)$ ou estações base LTE (eNodeB) das diversas tecnologias de acesso de rádio (WLAN/3G/ LTE, respetivamente). Esta arquitetura surge como relevante no contexto das tecnologias mais recentes de telecomunicações como o $5 \mathrm{G}$ e permite explorar a escala no 
contexto do IoT, da Internet Tátil ou da Internet de Mim. Para serviços virtualizados, a MEC traz vantagens como a redução de custos de implementação e a normalização dos procedimentos de gestão e orquestração.

\section{Configuração dos Cenários de Teste}

Para simular as condições de periferia de rede, foi configurado um cenário onde se implementou um servidor numa cloudlet instalada numa rede local. Optou-se pelas cloudlet uma vez que, na altura dos testes, se tratava da implementação mais avançada e mais estável em termos de desenvolvimento, e aquela que mais se aproximava do modelo idealizado pela ETSI. Para reproduzir o serviço da cloud, foi instalado um servidor numa plataforma AWS. O terminal do utilizador interliga-se por wi-fi com a infraestrutura de rede local e é através desta que acede a uma infraestrutura cablada de acesso à cloud.

Duas aplicações foram escolhidas que se assumem como sendo aplicações com fortes requisitos de computação e interação em tempo real. A primeira aplicação é o Fluid, uma aplicação de teste utilizada em computação gráfica interativa. A segunda aplicação é o FaceSwap, uma aplicação em tempo real de reconhecimento facial e troca de imagens. Foram realizados dois conjuntos de testes: um para comparar o efeito da latência dos cenários de cloudlet e de cloud no funcionamento das aplicações consideradas, e um outro para avaliar até que ponto poderá um aumento da capacidade de processamento no servidor da cloud minimizar o efeito da latência no funcionamento das aplicações testadas.

A Figura 1 apresenta a arquitetura de teste utilizada para avaliar o desempenho global das soluções cloudlet e cloud.

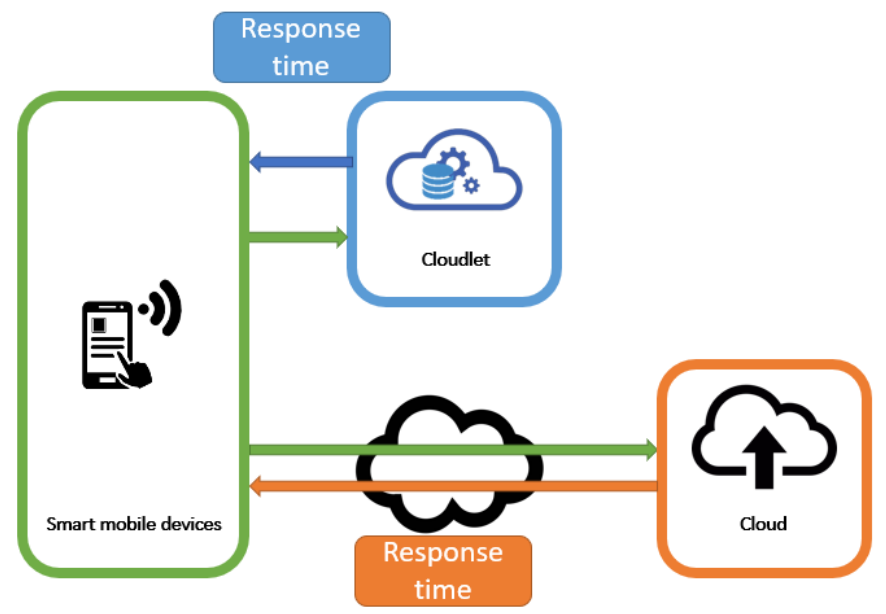

Figura 1 - Bancada de teste

A Tabela 1 resume as principais características dos sistemas utilizados para implementação da cloudlet e da cloud e do smartphone utilizados nos testes. Os dispositivos móveis foram ligados à mesma rede segundo a norma IEEE 802.11n. 


\begin{tabular}{ll}
\hline Equipamento & Características \\
\hline Cloudlet & Intel $^{\circledR}$ Core $^{\mathrm{TM}}$ i7 -2670 QM 2.20 GHz, 8 VCPUs \\
Satellite L755-1DR & 8 GB RAM, 30 GB Virtual Disk, 100 Mbps Ethernet \\
\hline Cloud & Intel+ ${ }^{\circledR}$ Xeon E5-2680 v2 2.80 GHz, 32 VCPUs \\
Amazon AWS-West & 32 GB RAM, 60 GB Virtual Disk, Amazon Enhanced Network \\
\hline Mobile Device & Exynos 8895 2.3 GHz Quad + 1.7 GHz Quad \\
Samsung S8 & 4 GB RAM, 64 GB Flash, 802.11a/b/g/n, WiFi \\
\hline
\end{tabular}

Tabela 1 - Configuração de Hardware

Para uma análise dos tempos de resposta conseguidos em cada cenário, os testes foram realizados com os servidores instalados em diferentes instâncias de máquinas virtuais na cloudlet e na cloud, conforme apresentado na Tabela 2.

Estes cenários permitiram a análise da influência do número de núcleos no desempenho da aplicação Fluid. Foram lançadas instâncias com 2 e 4 núcleos de CPU, na cloudlet e na cloud, e foi avaliado o desempenho em ambas as tecnologias. Para o efeito utilizaram-se diferentes instâncias de máquinas virtuais (Virtual Machines -VMs) na cloudlet e na cloud, tendo em conta que os servidores recomendados para a aplicação FaceSwap deveriam ter 4 núcleos e 8 GB de RAM (Wang, 2016). Foi considerada a variação do número de núcleos da CPU para permitir avaliar a influência da capacidade de processamento no tempo de resposta das aplicações. As instâncias foram iniciadas com 2, 4, 6 e 7 núcleos na cloudlet e com 1, 2, 4, 8, 16 e 32 núcleos na cloud. A mesma simulação foi realizada e os dados foram coletados para avaliar o desempenho as duas aplicações.

\begin{tabular}{lcc}
\hline $\mathbf{N}{ }^{\boldsymbol{o}} \boldsymbol{v}$ CPU/Instância & Cloudlet & Cloud \\
\hline 1 core & 1 CPU - 2 GB RAM & 1 CPU - 4 GB RAM \\
\hline 2 cores & 2 CPU - 4 GB RAM & 2 CPU - 8 GB RAM \\
\hline 4 cores & 4 CPU - 6 GB RAM & 4 CPU - 16 GB RAM \\
\hline 6 cores & 6 CPU - 5 GB RAM & \\
\hline 7 cores & 7 CPU - 6 GB RAM & \\
\hline 8 cores & & 8 CPU - 32 GB RAM \\
\hline 16 cores & & 16 CPU - 64 GB RAM \\
\hline 32 cores & & 32 CPU - 132 GB RAM \\
\hline
\end{tabular}

Tabela 2 - Configuração das instâncias

\subsection{Fluid}

Fluid é uma aplicação utilizada em demonstrações interativas de computação gráfica e um exemplo representativo de jogos em tempo real. É uma implementação simples de uma simulação de um fluido líquido, utilizando o método de Hidrodinâmica de Partículas Suavizadas (Smoothed-Particle Hydrodynamics - SPH) (Solenthaler \& Pajarola, 2009). O modelo SPH é um método lagrangiano usado para modelar o fluxo 
de um fluído, tratando cada partícula como um elemento discreto desse fluído. O Fluid transforma o ecrã do smartphone num recipiente onde as partículas se deslocam através dos movimentos provocados no dispositivo. O Fluid é dividido numa aplicação cliente executada no terminal móvel de um utilizador, e num servidor que executa a simulação dinâmica de cada uma das partículas do líquido.

A Figura 2 mostra as partículas a oscilar entre os lados do ecrã do smartphone com velocidade e direção diferentes devido à interação e movimentos do utilizador. Os dados do acelerómetro do smartphone são enviados para um mecanismo gráfico no servidor. Do lado do servidor, a aplicação executa um simulador físico baseado na hidrodinâmica de cada uma das partículas. O resultado da simulação é processado periodicamente e dá a ilusão de um líquido a oscilar dentro do ecrã do terminal móvel.

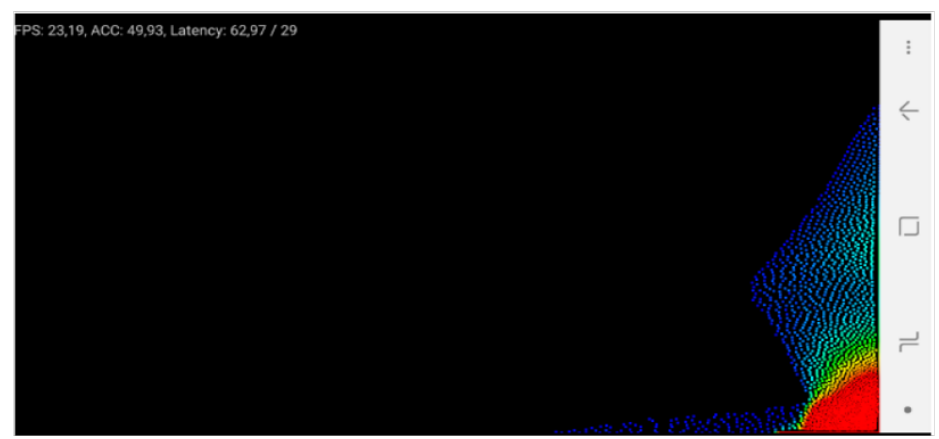

Figura 2 - Aplicação Fluid

Nesse cenário, o simulador executa o cálculo da trajetória de 2218 partículas com intervalos de tempo de 20ms, gerando até 50 Frames Por Segundo (FPS). A avaliação de desempenho mede o tempo de resposta do sistema utilizando as soluções de cloudlet e de cloud. Em ambos os casos, a medição começa quando o dispositivo envia os dados do sensor para o servidor e termina quando o servidor envia de volta a previsão estimada e essa é recebida no terminal. O ciclo de teste mede o tempo de resposta de várias iterações realizadas por um utilizador durante 5 minutos de interação, gerando 300 amostras. A experiência compara também os valores da taxa de FPS.

\subsection{FaceSwap}

O FaceSwap é uma aplicação Android que troca o rosto das pessoas em tempo real. O servidor do FaceSwap é composto por três camadas com base no rastreamento, deteção e reconhecimento de faces, conforme apresentado na Figura 3.

A aplicação cliente está constantemente a transmitir para o servidor imagens dos rostos no formato JPEG, com a dimensão 640x480. Do lado do servidor, se o reconhecimento do rosto for positivo, são enviadas caixas com as imagens dos rostos de substituição. A aplicação de reconhecimento de rosto detecta rostos nas imagens recebidas e tenta identificar o rosto a partir de uma base de dados pré-preenchida. As experiências realizadas consideram apenas a parte de reconhecimento num sistema 


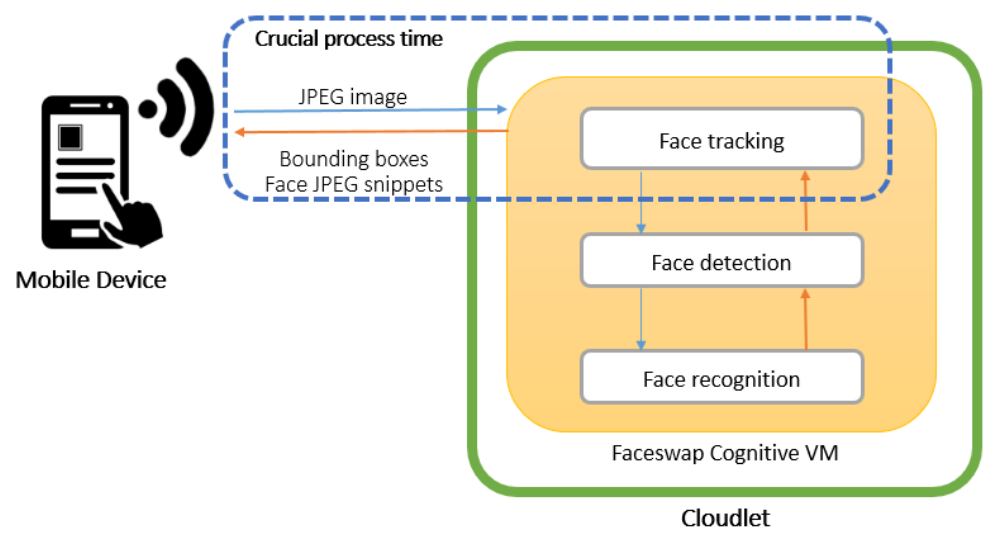

Figura 3 - Framework da aplicação cliente-servidor FaceSwap

treinado, possibilitando atingir tempos de resposta aceitáveis. Assim sendo, o treino dos classificadores e a população da base de dados são trabalhos feitos offline. Se as faces detectadas corresponderem às faces presentes na base de dados, uma resposta de comunicação bem-sucedida será enviada para permitir a troca das faces, conforme ilustrado na Figura 4.

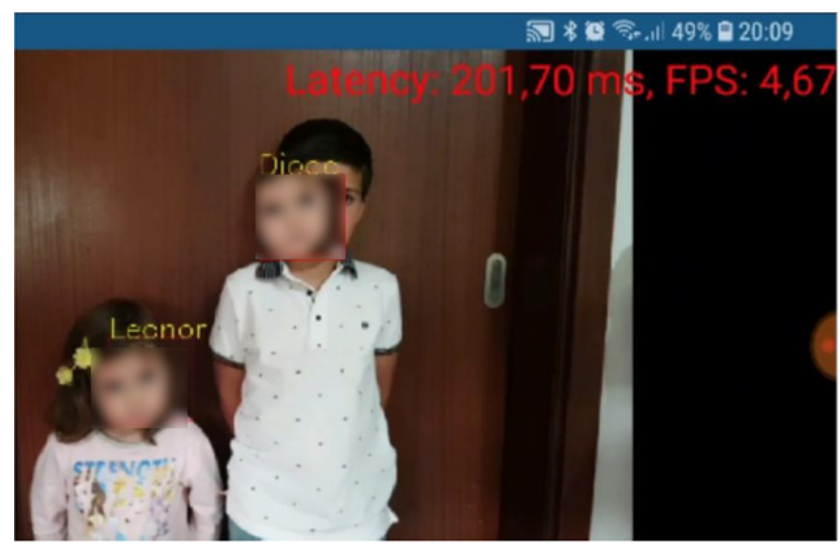

Figura 4 - Aplicação FaceSwap

Esta aplicação foi concebida para efetuar a troca de rostos de forma eficaz, pelo que um servidor FaceSwap deve ter pelo menos 4 núcleos e 8GB de RAM. Os testes do FaceSwap mostraram que a procura do rosto é executada em cerca de $15 \mathrm{~ms}$, sendo possível coletar dados a taxas elevadas de transferência de frames de imagens; e assim, obter uma resposta em tempo real. Por outro lado, como a deteção e o reconhecimento dos rostos demoram mais tempo, essas tarefas são efetuadas previamente (offline), permitindo que o objetivo principal ainda seja atingível. Quando os resultados ficam disponíveis, novas procuras podem ser iniciadas. 
A avaliação de desempenho mede o tempo de resposta do sistema utilizando a cloudlet em comparação com a cloud, isto é, a diferença de tempo que demora a troca entre a face substituída e a face original. O ciclo de teste possibilita a medição do tempo de resposta e da taxa de frames até chegar às 100 interações. No final de cada ciclo, foram registados os valores da latência e da taxa de FPS. O tempo de resposta determina a qualidade dos gráficos e deveria apresentar valores máximos de 200ms de forma a permitir usufruir de uma aplicação fluida; sendo que, quanto maior for, pior a experiência do utilizador.

\section{Resultados e Análise}

Das experiências realizadas foram obtidos e analisados os resultados. Dentre as plataformas AWS testadas (EC2-Europe, EC2-Asia, EC2-West e EC2-East) escolhida foi o EC2-West (situada em Oregon), uma vez que esta apresenta um tempo médio de ida e volta (Round Trip Time - RTT) de 101,5ms e disponibilizou as condições necessárias para a realização dos diferentes testes num contexto académico. A largura de banda média testada foi de $68 \mathrm{Mbps}$. As experiências avaliam a latência e a qualidade do utilizador implementando ciclos de interação que registam diferentes fenómenos que podem ocorrer em condições reais, como limitações de largura de banda, saturação de Wi-Fi e congestionamento, instabilidade de encaminhamento, falha do fornecedor de serviços. Esses ciclos permitem a apresentação e comparação dos resultados com um intervalo confiável sendo que estes resultados são apresentados utilizando a Função de Distribuição Cumulativa (Cumulative Distribution Function - CDF).

\subsection{Análise aos Resultados do Fluid}

A Figura 5 e a Figura 6 apresentam os resultados dos testes realizados à aplicação Fluid na cloudlet e na cloud. Um movimento fluído das partículas é obtido se a latência apresentar um valor máximo abaixo de 100ms. Se for superior, o movimento pode parecer mais lento, deteriorando a experiência do utilizador (Ha et al., 2013). Os valores obtidos no cenário da cloudlet são melhores, pois os valores medianos do tempo de resposta são de 32 a 34ms, comparativamente com os valores de $124 \mathrm{~ms}$ a $145 \mathrm{~ms}$ para a cloud, como ilustra a Figura 5 .

Na Figura 6 pode verificar-se que a taxa de frames apresenta valores medianos de 4447 FPS para a cloudlet e 18-22 FPS para a cloud. Como o servidor continua a gerar até 50 FPS e altera apenas os estados da simulação de acordo com os dados recebidos do acelerômetro, o cenário da cloudlet permite uma taxa de FPS duas vezes maior que a cloud. Os recursos obtidos permitem constatar que o uso da cloudlet pode ter um grande impacto reduzindo o tempo de resposta e aumentando substancialmente a qualidade da experiência do utilizador.

A análise de desempenho com o aumento dos recursos de CPU foi também considerada, através da execução de instâncias de VMs com maior capacidade de processamento (maior número de núcleos) e RAM. No entanto, verificamos que para o caso da aplicação Fluid não há uma diminuição significativa nos tempos de resposta, com 1 vCPU, 2 vCPUs ou 4 vCPUs. Para instâncias de VM na cloudlet com 1 vCPU e 4 vCPUs, verificamos que 
o tempo de resposta reduz cerca de $6 \%$ e aumenta da taxa de FPS em $4 \%$. Os valores obtidos na cloudlet cumprem com êxito os requisitos da aplicação.

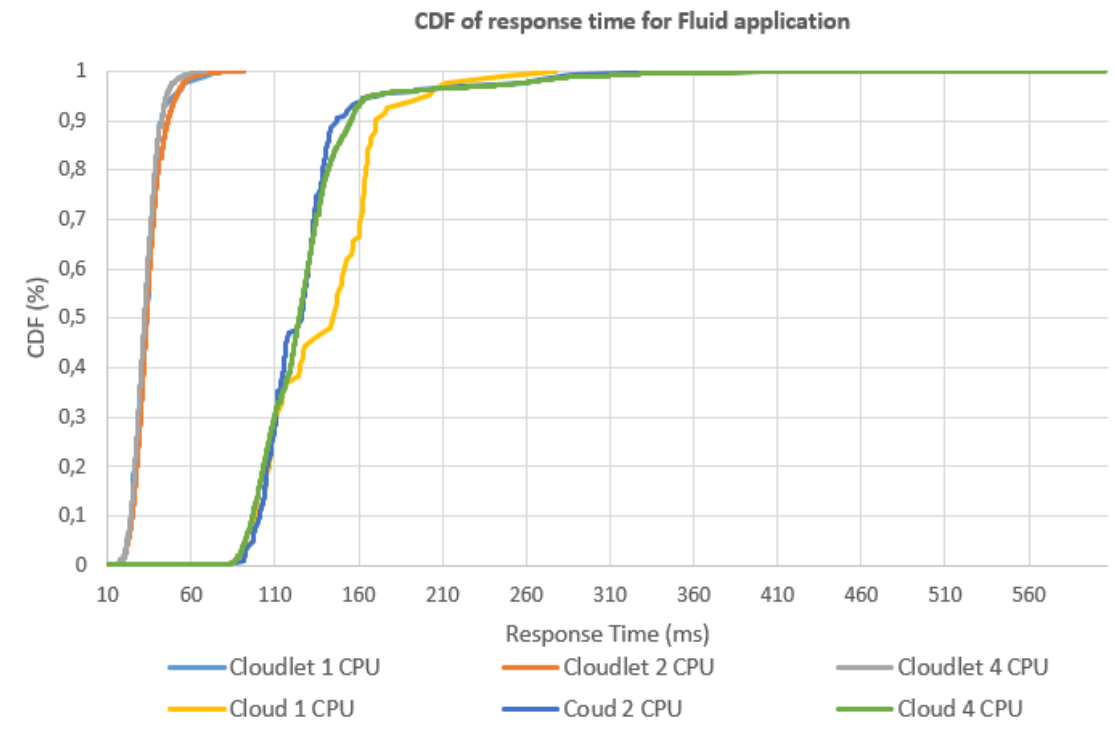

Figura 5 - Comparação dos tempos de resposta - Fluid

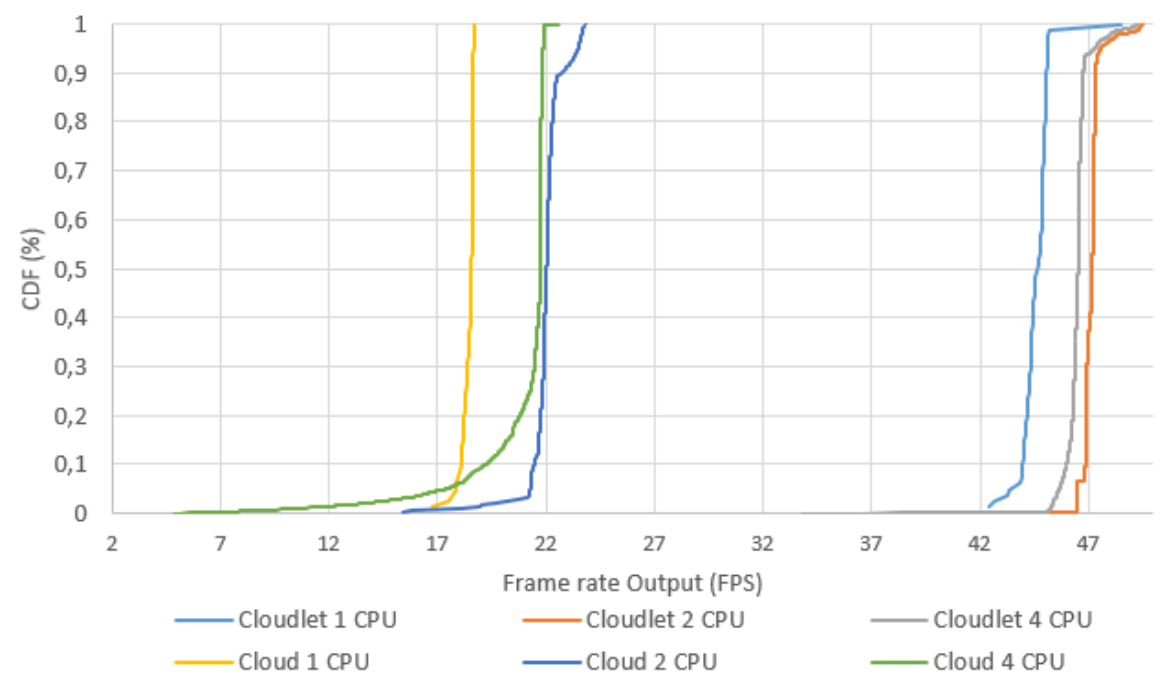

Figura 6 - Comparação das taxas de FPS - Fluid 


\subsection{Análise aos Resultados do FaceSwap}

Os testes realizados com o FaceSwap permitiram avaliar diretamente os valores do tempo de resposta e da taxa de FPS na cloudlet e na cloud (AWS EC2-Oregon) com instâncias de VMs com características aproximadas. Por um lado, a plataforma AWS apenas possibilita a utilização de instâncias com valores fixos de processamento (vCPU) e RAM. Por outro lado, as instâncias iniciadas na cloudlet apresentam limitações próprias do hardware utilizado. Portanto, não foi possível iniciar instâncias exatamente com a mesma capacidade de processamento (núcleos CPU) e de memória RAM. A experiência analisa também a melhoria do desempenho com o aumento da capacidade processamento das instâncias da VM.

O processo inicia-se com o lançamento das instâncias da VM do servidor FaceSwap. Depois disso, é iniciada a aplicação cliente FaceSwap no smartphone e selecionado o servidor da cloudlet. Note-se que os testes realizados pressupõem que em instâncias anteriores da cloudlet, foi executado o treino prévio da aplicação de reconhecimento de rostos e desse modo preenchida uma base de dados com os rostos. A seguir, é selecionada a funcionalidade de troca de rostos, rostos que são listados na base de dados previamente treinados e armazenados.

Na Figura 7 são apresentados os valores dos tempos de resposta da aplicação FaceSwap quando executada nos servidores da cloudlet e da cloud. Os valores obtidos permitem constatar que a performance no cenário cloudlet é consideravelmente superior, pois os valores medianos do tempo de resposta do cloudlet estão em torno de 38 a $43 \mathrm{~ms}$ e, para o cloud, os valores medianos estão entre os $205 \mathrm{~ms}$ e os $618 \mathrm{~ms}$. Para instâncias de VM com características semelhantes de processamento ( 2 e $4 \mathrm{CPU}$ ), os valores dos tempos de resposta da cloudlet são quase 5 vezes inferiores aos valores medidos na cloud (AWS EC2 Oregon).

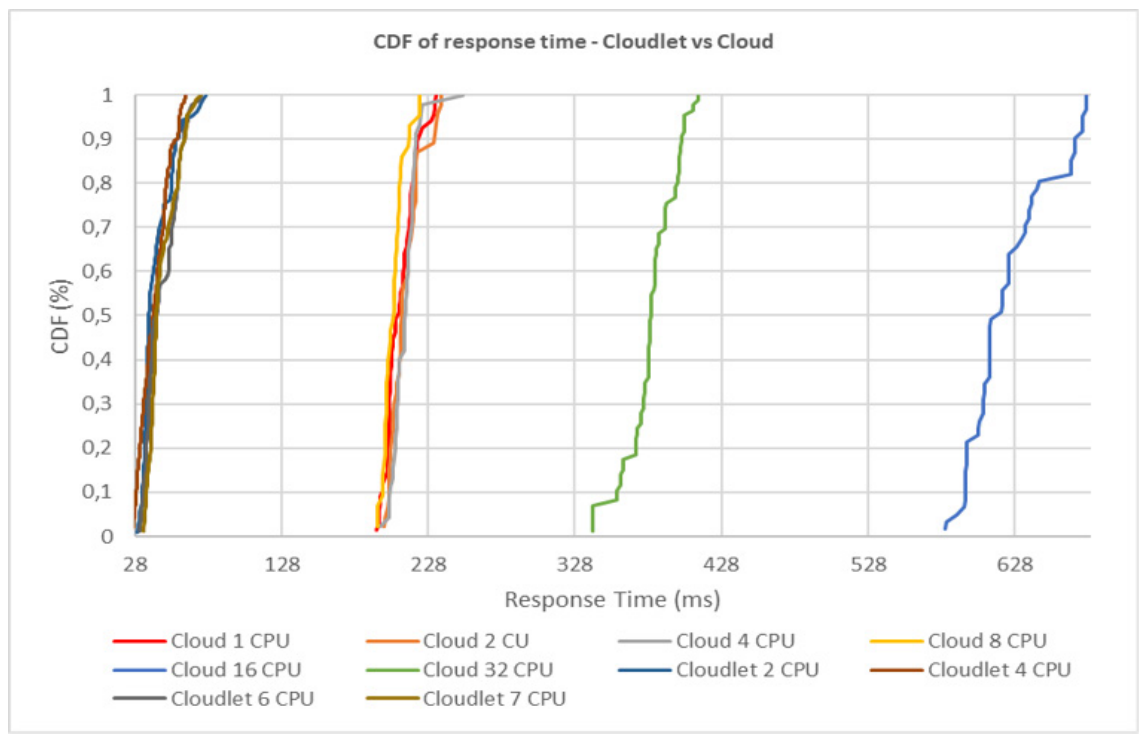

Figura 7 - Comparação dos tempos de resposta - FaceSwap 


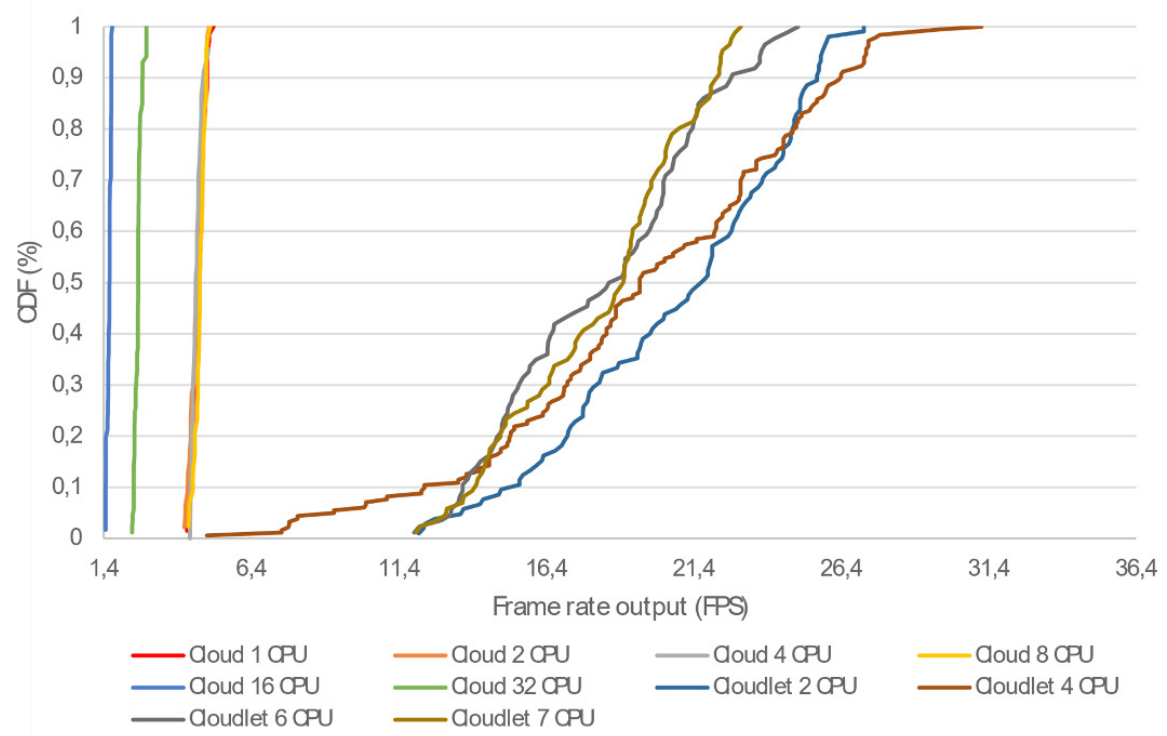

Figura 8 - Comparação das taxas de FPS - FaceSwap

Curiosamente, foi possível constatar que nos testes realizados com servidores de cloud com maior capacidade computacional, como nos sistemas de 16 e 32 vCPU, se assistiu a um aumento considerável no tempo de resposta da aplicação. Considerando que a capacidade de processamento foi aumentada, tal reflexo poderá estar associado a uma maior distância entre o cliente no smartphone e o servidor da cloud utilizado para a prestação do serviço alojamento com as características referidas. Repare-se, porém, que para os servidores FaceSwap de cloud com 16 vCPUs se obtiveram valores médios do tempo resposta em torno dos $600 \mathrm{~ms}$, e que o aumento do número de vCPUs para 32 permitiu reduzir de forma muito significativa esse valor para cerca de $360 \mathrm{~ms}$.

O tempo utilizado para o reconhecimento de rosto do servidor cloudlet é notoriamente inferior. A taxa de frames apresenta valores medianos de 19 a 22 FPS no cloudlet e de 2 a 5 FPS na cloud, como ilustra a Figura 8.

Nos testes realizados foi possível constatar que o uso da cloudlet garante uma taxa de FPS pelo menos 4 vezes maior do que a cloud, satisfazendo desta forma os requisitos de experiência do utilizador desta aplicação.

Da análise dos resultados obtidos foi possível constatar que estes estão em consonância com os obtidos nos testes realizados por J. Wang (Wang, 2016). Adicionalmente, foi efetuada uma comparação com outros trabalhos utilizando a aplicação FaceSwap (Ha et al., 2013) (Hu et al., 2016) (Chen et al., 2017), e nestes, os resultados obtidos nos testes realizados apresentam menor tempo de resposta nas soluções cloudlet. 


\section{Conclusões}

Este artigo apresenta um estudo de análise e comparação das tecnologias Mobile Edge Computing (MEC) e Mobile Cloud Computing (MCC) relativamente ao tempo de resposta de aplicações com fortes requisitos de processamento em tempo real em dispositivos móveis. Com este estudo pode concluir-se que o MEC pode assumir um papel preponderante ao complementar o funcionamento das soluções assentes na cloud (MCC).

Em aplicações com requisitos de processamento intensivo em tempo real, a parte sensível ao atraso do aplicativo pode ser executada no servidor MEC, enquanto a parte intensiva, mais tolerante a atraso do aplicativo pode ser executada no servidor na cloud. O MEC permite que os dispositivos móveis conectados executem aplicativos de processamento intensivos e de tempo real na periferia da rede, libertando os terminais dos clientes utilizadores dessas tarefas. Para além de que, a maior proximidade entre servidor e dispositivo cliente permite reduzir significativamente o tempo de resposta das aplicações e, deste modo, melhorar a experiência do utilizador.

Este artigo apresenta os resultados de comparação de implementações do MEC com o MCC recorrendo a duas aplicações com requisitos de processamento em tempo real, o Fluid e o FaceSwap.

Dos resultados obtidos, mostra-se que o MEC com a implementação via cloudlet permite melhorar a qualidade e a experiência do utilizador. Os valores dos tempos de resposta medidos na aplicação Fluid fixam-se sempre abaixo de $100 \mathrm{~ms}$, sendo aproximadamente quatro vezes inferiores aos valores apresentados utilizando a cloud. Além disso, pela utilização da cloudlet, o movimento das partículas é mais fluido e não se degrada facilmente como na cloud.

Os valores medidos na utilização do FaceSwap apresentam um tempo de resposta cerca de cinco vezes inferior, e sempre abaixo dos 10oms, quando comparada com os valores de uma implementação de cloud. Relativamente à taxa de FPS de imagens, o cloudlet apresenta valores quatro vezes mais elevados do que na cloud, o que evidencia a melhoria da qualidade de experiência do utilizador.

Em suma, quando comparado aos servidores remotos na cloud, verifica-se que, nas aplicações com as características selecionadas, o MEC permite menor tempo de resposta e uma maior taxa de frames do que o MCC. Os resultados permitem concluir que a arquitetura MEC proporciona um melhor desempenho e melhor experiência ao utilizador, quando comparada com a cloud.

\section{Referências}

Aazam, M., \& Huh, E.N. (2014). Fog computing and smart gateway-based communication for cloud of things. In 2014 International Conference on Future Internet of Things and Cloud, (pp. 464-470). doi: 10.1109/FiCloud.2014.83.

Abolfazli, S., Sanaei, Z., Ahmed, E., Gani, A., \& Buyya, R. (2013). Cloud-based augmentation for mobile devices: motivation, taxonomies, and open challenges. IEEE Communications Surveys \& Tutorials, 16(1), 337-368. doi: 10.1109/ SURV.2013.070813.00285 
Achanta, V., Sureshbabu, N., Thomas, V., Sahitya, M., \& Rao, S. (2012). Cloudletbased multilingual dictionaries. In Third International Conference on Services in Emerging Markets, (pp. 30-36). doi: 10.1109/ICSEM.2012.12

Ai, Y., Peng, M., \& Zhang, K. (2017). Edge cloud computing technologies for internet of things: A primer. doi: 10.1016/j.dcan.2017.07.001

Araújo, V. M., Cota, M. P. (2016). Software como um Serviço: uma visão holística. RISTI Revista Ibérica de Sistemas e Tecnologias de Informação, (19), 145-157. doi: 10.17013/risti.19.145-157

Armbrust, M., Fox, A., Griffith, R., Joseph, A., Katz, R., Konwinski, A., Lee, G., \& Stoica, I. (2009). Above the clouds: A berkeley view of cloud computing. Berkeley: Dept Electrical Eng and Computer Sciences, University of California.

Beck, M. T., Werner, M., Feld, S., \& Schimper, S. (2014). Mobile edge computing: A taxonomy. In Proceedings of the Sixth International Conference on Advances in Future Internet, (pp. 48-55).

Blockstrand, M., Holm, T., Kling, L.-O., Kog, R., \& Wallin, B. (2011). More than 50 billion connected devices - taking connected devices to mass market and profitability. Retrieved from: https://www.akos-rs.si/files/Telekomunikacije/Digitalna_ agenda/Internetni_protokol_Ipv6/More-than-50-billion-connected-devices.pdf

Bonomi, F., Milito, R., Natarajan, P., \& Zhu, J. (2014). Fog computing: A platform for internet of things and analytics. In Big data and internet of things: A roadmap for smart environments, (pp. 169-186). Cham: Springer International Publishing. doi: 10.1007/978-3-319-05029-4_7.

Bonomi, F., Milito, R., Zhu, J., \& Addepalli, S. (2012). Fog computing and its role in the internet of things. In Proceedings of the first edition of the MCC workshop on Mobile cloud computing, (pp. 13-16). ACM. doi: 10.1145/2342509.2342513

Chen, Z., Hu, W., Wang, J., Zhao, S., Amos, B., Wu, G., \& Satyanarayanan, M. (2017). An Empirical Study of Latency in an Emerging Class of Edge Computing Applications for Wearable Cognitive Assistance. In Proceeding of the SecondACM/IEEESymposium on Edge Computing, (pp. 14:1-14:14). ACM. doi: 10.1145/3132211.3134458

Dinh, H. T., Lee, C., Niyato, D., \& Wang, P. (2013). A survey of mobile cloud computing: architecture, applications, and approaches. Wireless communications and mobile computing, 13(18), 1587-1611. doi: 10.1002/wcm.1203

Elijah, G. (2009). Cloudlet-based Edge Computing. Retrieved from: http://elijah. cs.cmu.edu/.

ETSI. (2014). Mobile-Edge Computing. Introductory Technical White Paper. https://portal.etsi.org/Portals/o/TBpages/MEC/Docs/Mobile-edge_ Computing_-_Introductory_Technical_White_Paper_V1\%2018-09-14.pdf

ETSI. (2015). Mobile Edge Computing (MEC) Service Scenarios V1.1.1. 
Fortes, N., Pereira, J. H., \& Costa, J. F. da. (2016). A adoção de serviços cloud computing pelas empresas portuguesas: O papel dos esforços de marketing. RISTI - Revista Ibérica de Sistemas e Tecnologias de Informação, (18), 33-48. DOI: https://dx.doi. org/10.17013/risti.18.33-48

GSMA Intelligence, (2014). Understanding 5G: Perspectives on future technological advancements in mobile. White paper, 1-26.

Ha, K., Pillai, P., Lewis, G., Simanta, S., Clinch, S., Davies, N., \& Satyanarayanan, M. (2013). The impact of mobile multimedia applications on data center consolidation. In 2013 IEEE international conference on cloud engineering (IC2E), (pp. 166-176). doi: 10.1109/IC2E.2013.17

Hu, W., Gao, Y., Ha, K., Wang, J., Amos, B., Chen, Z., Pillai, P., \& Satyanarayanan, M. (2016). Quantifying the Impact of Edge Computing on Mobile Applications. In Proceedings of the 7th ACM SIGOPS Asia-Pacific Workshop on Systems, (pp. 5:1-5:8). ACM. doi: 10.1145/2967360.2967369

Khan, K., Wang, Q., Luo, C., Wang, X., \& Grecos, C. (2014). Comparative study of internet cloud and cloudlet over wireless mesh networks for real-time applications. In Real-Time Image and Video Processing 2014, (pp.91390K). doi: 10.1117/12.2052474

Klas, G. (2016). Fog computing and mobile edge cloud gain momentum open fog consortium, etsi mec and cloudlets. In SoftNet 2016 Conference, August 21, 2016, Rome.

Koukoumidis, E., Lymberopoulos, D., Strauss, K., Liu, J., \& Burger, D. (2011). Pocket cloudlets. In ACM SIGARCH Computer Architecture News, (pp. 71-184). doi: 10.1145/2248487.1950387

Mao, Y., You, C., Zhang, J., Huang, K., \& Letaief, K. B. (2017). A survey on mobile edge computing: The communication perspective. IEEE Communications Surveys \& Tutorials, 19(4), 2322-2358. doi: 10.1109/COMST.2017.2745201

Patel, M., Naughton, B., Chan, C., Sprecher, N., Abeta, S., \& Neal, A. (2014). Mobileedge computing introductory technical white paper. White paper, mobile-edge computing (MEC) industry initiative, (pp. 1089-7801).

Qing, W., Zheng, H., Ming, W., \& Haifeng, L. (2013). Cactse: Cloudlet aided cooperative terminals service environment for mobile proximity Content delivery. China Communications, 10(6), 47-59. doi: 10.1109/CC.2013.6549258.

Satyanarayanan, M. (2013). Cloudlets: At the Leading Edge of Cloud-mobile Convergence. In Proceedings of the 9th International ACM Sigsoft Conference on Quality of Software Architectures (QoSA '13), (pp. 1-2). doi: 10.1145/2465478.2465494

Satyanarayanan, M., Bahl, P., Caceres, R., \& Davies, N. (2009). The Case for VMBased Cloudlets in Mobile Computing IEEE Pervasive Computing, 8(4), 14-23. doi: 10.1109/MPRV.2009.82 
Shahzadi, S., Iqbal, M., Dagiuklas, T., \& Qayyum, Z. (2017). Multi-access edge computing: open issues, challenges and future perspectives. Journal of Cloud Computing, 6(1), 30. doi: 10.1186/s13677-017-0097-9

Shenoy, K., Bhokare, P., \& Pai, U. (2015). Fog computing future of cloud computing, International Journal of Science and Research, 4(6), 55-56.

Simanta, S., Ha, K., Lewis, G., Morris, E., \& Satyanarayanan, M. (2012). A Reference Architecture for Mobile Code Offload in Hostile Environments. In Joint Working IEEE/IFIP Conference on Software Architecture and European Conference on Software Architecture, Helsinki, 2012, (pp. 282-286). doi: 10.1109/WICSAECSA.212.46.

Skarpness, S. (2017). Keynote: Beyond the Cloud: Edge Computing. Recuperado de: https://www.youtube.com/watch?v=mCDXnls6pQk

Solenthaler, B., \& Pajarola, R. (2009). Predictive-corrective Incompressible SPH. ACM SIGGRAPH 2009 papers, 28(3), 40:1-40:6. doi: 10.1145/1576246.1531346

Soyata, T., Muraleedharan, R., Funai, C., Kwon, M., \& Heinzelman, W. (2012). Cloudvision: Real-time face recognition using a mobile-cloudlet-cloud acceleration architecture. In 2012, IEEE symposium on computers and communications (ISCC), (pp.000059-000066). doi: 10.1109/ISCC.2012.6249269

Suryawanshi, R., \& Mandlik, G. (2015). Focusing on mobile users at edge and internet of things using fog computing. Int. J Sci. Eng. Tech. Res, 4(17), 3225-3231.

Verbelen, T., Simoens, P., De Turck, F., \& Dhoedt, B. (2012). Cloudlets: Bringing the cloud to the mobile user. In Proceedings of the third ACM workshop on Mobile cloud computing and services, (pp. 29-36). ACM. doi: 10.1145/2307849.2307858

Wang, J. (2016). FaceSwap (Internet) Retrieved from: https://cmusatyalab. github.io/ faceswap/dev-guide/

Zhang, Q., Cheng, L., \& Boutaba, R. (2010). Cloud computing: state-of-the-art and research challenges. Journal of internet services and applications, 1(1), 7-18. doi: 10.1007/s13174-010-0007-6 